\title{
Deterioration of pre-existing hemiparesis brought about by subsequent ipsilateral lacunar infarction
}

\author{
T Ago, T Kitazono, H Ooboshi, J Takada, T Yoshiura, F Mihara, S Ibayashi, M lida
}

J Neurol Neurosurg Psychiatry 2003;74:1 152-1153

\begin{abstract}
Mechanisms of post-stroke recovery are still poorly understood. Recent evidence suggests that cortical reorganisation in the unaffected hemisphere plays an important role. A 59 year old man developed a small lacunar infarct in the left corona radiata, which then caused marked deterioration in a pre-existing left hemiparesis that had resulted from an earlier right putaminal haemorrhage. Functional magnetic resonance imaging showed that the paretic left hand grip activated the ipsilateral left motor areas, but not the right hemispheric motor areas. This suggests that partial recovery of the left hemiparesis had been brought about by cortical reorganisation of the left hemisphere and intensification of the uncrossed corticospinal tract. The subsequent small infarct may have damaged the uncrossed tract, thereby causing the pre-existing hemiparesis to deteriorate even further.
\end{abstract}

$\mathrm{P}$ artial or complete recovery of motor function is the general rule after stroke. However, the mechanisms of post-stroke recovery are still poorly understood. ${ }^{1}$ We report a case where cortical reorganisation of the unaffected hemisphere may have played a significant role in post-stroke recovery, but where this partial recovery of hemiparesis appears to have been damaged by a subsequent small ipsilateral infarct in the hitherto unaffected hemisphere.

\section{CASE REPORT}

A 59 year old man was admitted to our hospital because of marked deterioration in pre-existing left hemiparesis. The patient was an outpatient with a history of right putaminal haemorrhage five years earlier, since when he had had a mild left hemiparesis. Upon admission, the patient was alert. His blood pressure was $134 / 70 \mathrm{mmHg}$ and his pulse rate was 70 beats/min. Neurological examination showed that the preexisting left hemiparesis had deteriorated markedly, but that the function of his face and limbs on the right side remained intact. Cranial magnetic resonance fluid attenuated inversion recovery (FLAIR) imaging showed a small high intensity area in the left corona radiata (fig lA), which was shown to be a fresh lesion by diffusion weighted imaging (fig 1B). As no stenotic lesions of the large carotid or cerebral arteries were demonstrated by magnetic resonance angiography or cervical ultrasound sonography (findings not presented), the new lesion was diagnosed as a lacunar infarct. A functional magnetic resonance imaging (fMRI) study-in which the patient was scanned while doing a simple motor task consisting of five repetitions of self paced hand grip for 40 seconds followed by rest for 40 seconds-showed that the grip of the non-paretic right hand activated the contralateral left sensorimotor cortex (fig $\mathrm{lC}$, upper row). On the other hand, grip of the paretic left hand also increased the cerebral blood flow in the ipsilateral larger areas including the sensorimotor cortex, the supplementary motor area, and the premotor cortex, but not in any of the motor areas of the contralateral hemisphere (fig 1C, lower row).

\section{DISCUSSION}

We describe a case where a small lacunar infarct in the left corona radiata caused deterioration in a pre-existing left hemiparesis that had resulted from an earlier right putaminal haemorrhage.

To elucidate this enigmatic phenomenon, we employed fMRI. The fMRI study clearly showed that a significant shift in left motor control to the ipsilateral cortex had taken place after the first stroke. It appears that cortical reorganisation of the left hemispheric motor areas had occurred, thereby contributing to the partial recovery of the left hemiparesis in this patient (fig lC, lower row).

Various studies, including positron emission tomography ${ }^{2-4}$ and fMRI, ${ }^{56}$ have identified three processes related to post-stroke motor recovery ${ }^{1}$ : activation of the peri-infarction area, $^{57}$ increased reliance on motor areas such as the supplementary motor area and the premotor cortex ${ }^{48}$ and cortical reorganisation within the motor areas of the unaffected hemisphere. ${ }^{4-6910}$ The present case seems to have involved the third process.

In this case, a small lesion in the left corona radiata manifestly caused deterioration in the pre-existing left hemiparesis; however, it did not produce any right motor dysfunction. A possible explanation for the absence of right motor dysfunction may be that the lesion was too small to produce damage to the crossed corticospinal tract. Indeed, a small infarct in the corona radiata is often asymptomatic. On the other hand, it may simply be that by chance the infarct produced direct damage to the uncrossed corticospinal tract itself.

When an initial stroke results in extensive damage to motor function, a subsequent stroke contralateral to the first stroke sometimes causes deterioration in the ipsilateral pre-existing hemiparesis, as well as producing contralateral motor dysfunction. Cortical reorganisation in the unaffected hemisphere after the first stroke may be involved in the occurrence of such symptoms. fMRI may be useful for evaluating the pathophysiology of these patients. Further cases are necessary to confirm our interpretation of the interesting phenomenon seen in this case. 

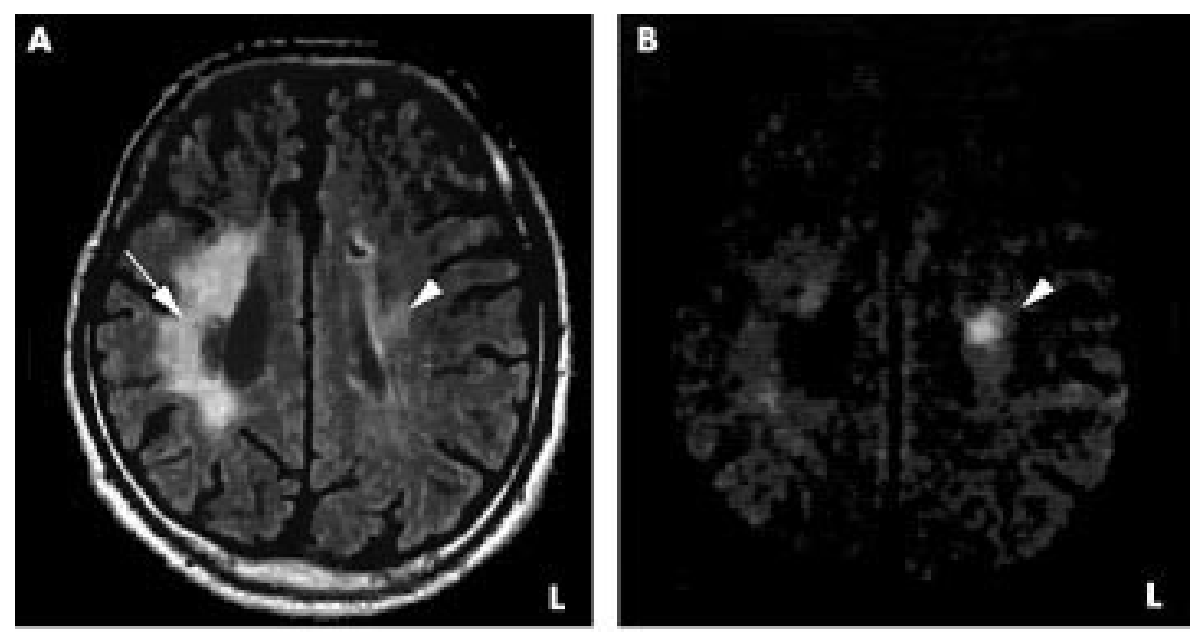

Figure 1 (A) FLAIR and (B) diffusion weighted imaging (b-factor 1000) on the second day of hospital admission revealed a small high intensity area in the left corona radiata (arrowhead), suggesting a fresh lacunar infarct. FLAIR imaging showed a large subcortical high intensity area which represented an old putaminal haemorrhage (arrow). (C) FMRI induced by the grip task is shown. Arrowheads point to areas activated by hand grip. Upper row: right hand grip activated the contralateral left sensorimotor cortex. Lower row: left paretic hand grip activated the ipsilateral left sensorimotor cortex, supplementary motor area, and premotor cortex. fMRI, functional magnetic resonance C Right hand grip imaging.
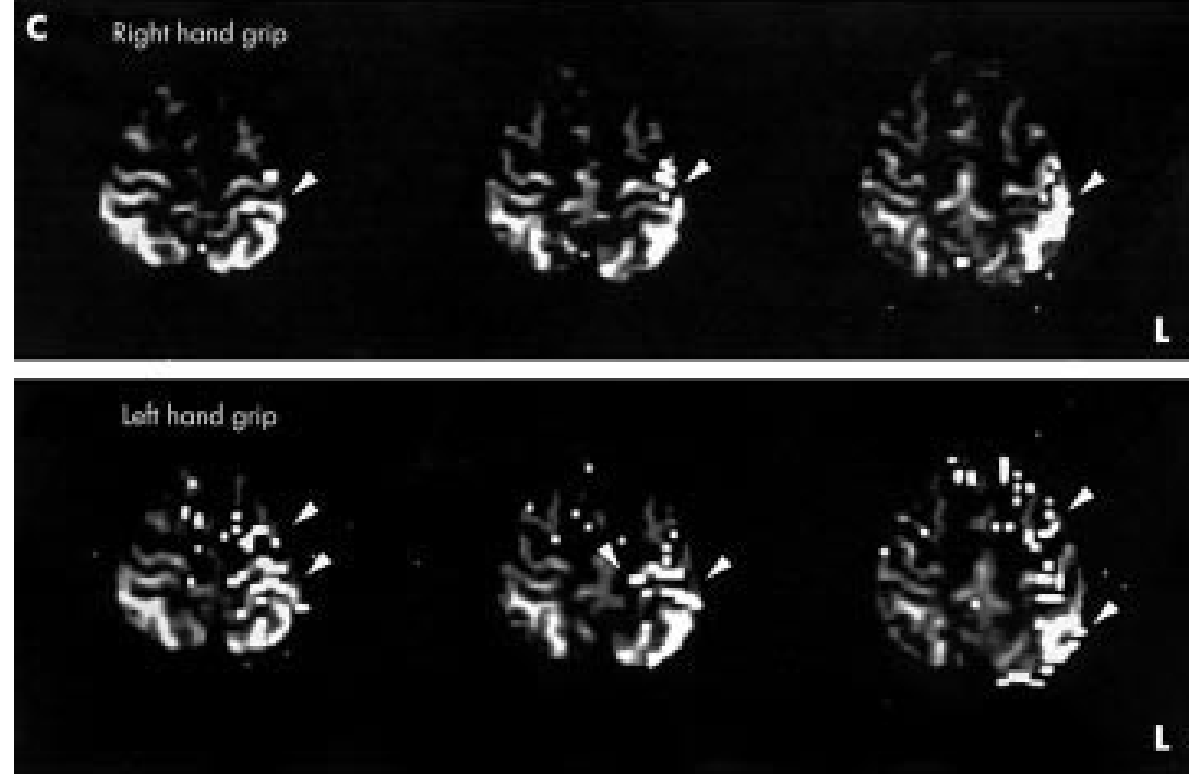

\section{Authors' affiliations}

T Ago, T Kitazono, H Ooboshi, J Takada, S Ibayashi, M lida Department of Medicine and Clinical Science, Graduate School of Medical Sciences, Kyushu University, Fukuoka, Japan

T Yoshiura, F Mihara, Department of Clinical Radiology, Graduate School of Medical Sciences, Kyushu University,

Correspondence to: Dr Tetsuro Ago, Department of Medicine and Clinical Science, Graduate School of Medical Sciences, Kyushu University, Maidashi 3-1-1, Higashi-ku, Fukuoka 812-8582, Japan; agou@intmed2.med.kyushu-u.ac.jp

Received 11 December 2002

In revised form 15 April 2003

Accepted 15 April 2003

\section{REFERENCES}

1 Johansson BB. Brain plasticity and stroke rehabilitation. The Willis lecture. Stroke 2000;31:223-30.

2 Weiller C, Chollet F, Friston KJ, et al. Functional reorganization of the brain in recovery from striatocapsular infarction in man. Ann Neurol 1992;31:463-72.
3 Chollet F, DiPiero V, Wise RJ, et al. The functional anatomy of motor recovery after stroke in humans: a study with positron emission tomography. Ann Neurol 1991;29:63-71.

4 Nelles G, Spiekermann G, Jueptner M, et al. Reorganisation of sensory and motor systems in hemiplegic stroke patients. A positron emission tomography study. Stroke 1999;30:1510-16.

5 Cramer SC, Nelles G, Benson RR, et al. A functional MRI study of subjects recovered from hemiparetic stroke. Stroke 1997;28:2518-27.

6 Cao Y, D'Olhaberriague L, Vikingstad EM, et al. Pilot study of functional MRI to assess cerebral activation of motor function after poststroke hemiparesis. Stroke 1998;29:112-22.

7 Nudo RJ, Wise BM, SiFuentes F, et al. Neural substrates for the effects of rehabilitative training on motor recovery after ischemic infarct. Science 1996;272:1791-4

8 Aizawa $\mathbf{H}$, Inase $M$, Mushiake $\mathrm{H}$, et al. Reorganization of activity in the supplementary motor area associated with motor learning and functional recovery. Exp Brain Res 1991;84:668-71.

9 Jones TA, Schallert T. Use-dependent growth of pyramidal neurons after neocortical damage. J Neurosci 1994; 14:2140-52.

10 Green JB, Bialy Y, Sora E, Ricamato A. High-resolution EEG in poststroke hemiparesis can identify ipsilateral generators during motor tasks. Stroke 1999;30:2659-65. 